\title{
Screening History in Vaginal Precancer and Cancer: A Retrospective Study of 2131 Cases in China
}

\section{Lu Zhang \\ Qing Wang \\ Hongwei Zhang \\ Yu Xie (D) \\ Long Sui (D) \\ Qing Cong}

Medical Center of Diagnosis and Treatment for Cervical Diseases, Obstetrics and Gynecology Hospital of Fudan University, Shanghai, People's Republic of China
Correspondence: Qing Cong; Long Sui Medical Center of Diagnosis and Treatment for Cervical Diseases, Obstetrics and Gynecology Hospital of Fudan University, Shanghai, People's Republic of China

Tel +86 I5900798278; +86 I380I788II8

Fax +862133189900

Email151468770@qq.com;

suilong@fudan.edu.cn
Purpose: To examine the screening history of vaginal intraepithelial neoplasia (VaIN) and vaginal cancer.

Patients and Methods: We included women with histologically confirmed VaIN or vaginal cancer by colposcopy-directed biopsy between 1 January 2019 and 31 December 2019. The results of cytology, hrHPV, colposcopic examination and history of hysterectomy were retrospectively analysed.

Results: A total of 26,432 colposcopies were performed during the study period, among which 2131 women (1835 [86.1\%] with VaIN 1; 268 [12.6\%] with VaIN 2/3; and 28 [1.3\%] with vaginal cancer) were retrospectively studied. hrHPV test positivity was significantly higher than that of cytology for VaIN 1 (84.4\% vs 67.3\%; P < 0.001) and VaIN 2/3 (92.0\% vs $79.9 \%$; $\mathrm{P}<0.001)$ but not for vaginal cancer $(84.6 \%$ vs $78.6 \% ; \mathrm{P}=0.73)$. Additionally, the concordance rates for colposcopic impression were $79.5 \%, 54.5 \%$, and $92.8 \%$ for VaIN1, VaIN2/3, and vaginal cancer, respectively. All 372 patients had a history of hysterectomy, and $81.0 \%(282 / 348)$ of indications were related to cervical precancer and cancer. Although cytology test positivity was significantly higher in patients with hysterectomy than in patients without hysterectomy $(76.2 \%$ vs $67.5 \%$; P < 0.001), cytology combined with hrHPV can help to detect more than $95 \%$ of VaIN and vaginal cancer cases in both groups $(96.2 \%$ for patients with hysterectomy and $96.5 \%$ for patients without hysterectomy).

Conclusion: VaIN and vaginal cancer are not rare diseases. Although cytology was sensitive $(67.5 \%-76.2 \%)$ for detecting vaginal lesions regardless of hysterectomy, cytology combined with hrHPV improves detection accuracy up to $95 \%$ in both groups.

Keywords: cytology, human papilloma virus, colposcopy, vaginal intraepithelial neoplasia, vaginal cancer

\section{Introduction}

Vaginal intraepithelial neoplasia (VaIN) and vaginal cancer are caused by human papilloma virus (HPV) infection of the vagina. ${ }^{1-4}$ VaIN 2/3 is a precancerous lesion that is analogous to cervical intraepithelial neoplasia (CIN) 2/3, whereas VaIN 1 is the benign vaginal manifestation of HPV infection. ${ }^{5,6}$ Other known risk factors for VaIN and vaginal cancer include immunosuppression, smoking, multiple intercourse partners, and early coitarche. ${ }^{7,8}$ Additionally, a history of cervical lesions and previous hysterectomy for HPV-related cervical diseases are other major risk factors. ${ }^{9,10}$

The incidence of VaIN has been increasing steadily because of widespread cytology with high-risk HPV (hrHPV) cotesting and colposcopy. At the largest obstetrics 
and gynecology tertiary teaching hospital in China, the mean detection rate of VaIN in all lower genital tract intraepithelial lesions was reportedly $11.49 \% \quad(1923 / 16,732)$, which increased from $8.09 \%$ in 2013 to $13.08 \%$ in $2015 .^{11}$ However, most vaginal lesions are diagnosed during cervical cancer screening, and there are no guidelines for specifically conducting screening for vaginal lesions. In addition, cytology and hrHPV tests were performed above the surface of the cervix. Little is known about whether cervical screening is the appropriate clinical choice for the detection of vaginal lesions. $^{3,12-16}$ Because of the reasons above, we aimed to explore the roles of cervical screening history, including cytology, hrHPV genotyping, and colposcopic examinations, in colposcopy-directed biopsy (CDB)-confirmed VaIN and vaginal cancer $(\mathrm{VaIN}+)$.

\section{Patients and Methods}

\section{Study Population}

As the largest OBGYN referral centre in China, we admit VaIN and vaginal cancer patients across the country. The inclusion criterion included women visiting our hospital in Yangpu District between 1 January 2019 and 31 December 2019. VaIN or vaginal cancers were histologically diagnosed under CDB and either performed in our hospital or by a referring institution. Any cervical cancer case with vaginal invasion was excluded. For repeat patients, we chose the first time they underwent CDB during this period.

Permission was granted by the institutional review board of the Obstetrics and Gynecology Hospital of Fudan University before data extraction was performed. All available data, including age, history of surgery, cytology results, hrHPV test results, colposcopic examination results, and pathology results, were extracted from medical records. For patients referred to our hospital, original pathologic specimens were re-examined by a senior pathologist, and colposcopy was performed by a senior colposcopist to revalidate the presence or absence of invasive lesions, with subsequent biopsies performed for areas suspected of neoplasia.

\section{Cytology Screening Testing}

A liquid-based Pap test (SurePathTM; Becton, Dickinson, Franklin Lakes, NJ, USA) was used for cytology testing. The results, including negative for intraepithelial lesion or malignancy (NILM), atypical squamous cells of undetermined significance (ASC-US), low-grade squamous intraepithelial lesion (LSIL), atypical squamous cells that cannot exclude HSIL (ASC-H), high-grade squamous intraepithelial lesion (HSIL), atypical glandular cells (AGC), and squamous cell carcinoma (SCC), were reported using the Bethesda System.

\section{hrHPV DNA Screening}

hrHPV testing was performed using hybrid capture- 2 testing (Digene, Maryland, USA), the Cobas 4800 assay (Roche, Penzberg, Germany) or a fluorescence-based multiplex real-time HPV DNA genotyping kit (Bioperfectus, Jiangsu, China), which can detect high-risk HPV types 16, $18,31,33,35,39,45,51,52,56,58,59,66$, and 68 .

\section{Colposcopic Examination}

Cytology/hrHPV testing was performed at the clinic within 6 weeks before colposcopy. Oestrogen was applied prior to colposcopic examinations among postmenopausal women. The indications for colposcopy at our hospital were as follows: abnormal cytology results (ASCUS, LSIL, HSIL, ASC-H, AGC, SCC); positive hrHPV results; symptoms or signs of possible cervical/vaginal/vulvar cancer, including any suspicious cervical abnormality found during pelvic examination, abnormal genital tract bleeding, or unexplained cervicovaginal discharge; first follow-up after cervical conization; and first follow-up after hysterectomy indicated for cervical cancer or HSIL. Colposcopic examinations and diagnoses were performed by experienced colposcopists following the 2011 International Federation of Cervical Pathology and Colposcopy terminology. ${ }^{17}$ A detailed description of colposcopic findings and initial diagnosis were recorded based on our previous study: ${ }^{18} 1$ ) General assessment: adequate or inadequate; 2) Normal colposcopic findings: mature or atrophic squamous epithelium; 3) Abnormal colposcopic findings: the location (anterior, posterior, or lateral) and total size of the lesion; the maximum size of single lesion and the number of lesions; Grade 1 (minor) including thin acetowhite epithelium, fine punctuation and fine mosaic; Grade 2 (major) including dense acetowhite epithelium, coarse punctuation and coarse mosaic; suspicious for invasion including atypical vessels, fragile vessels, and an irregular surface; and nonspecific findings including columnar epithelium (adenosis); 4) Miscellaneous findings: erosion, condyloma, polyp, cyst, endometriosis, etc; 5) Finally, colposcopic impression was reckoned, which was classified as normal or benign lesion, VaIN 1, VaIN 2/3, or suspicion of cancer. Based on the application of $5 \%$ acetic solution and Lugol's iodine 
solution, targeted biopsies of suspected cervical, vaginal, and vulvar lesions were performed. For patients after hysterectomy, we specifically inspected the 2 ends of the vault suture line to avoid hidden vaginal lesions in vaginal dimples.

\section{Statistical Analysis}

Data calculation was performed using SPSS version 24.0 (IBM, Armonk, NY, USA). Normally distributed continuous variables are expressed as the mean $\pm \mathrm{SD}$, and nonnormally distributed continuous variables are expressed as the median (interquartile range, IQR). Categorical variables are presented as numbers and proportions. The Kruskal-Wallis test was used to compare continuous variables, whereas the chi-square or Fisher's exact tests were used for categorical variables. A two-tailed P-value $<0.05$ was deemed statistically significant.

The parameters listed below were calculated for the HPV16, HPV18 and non16/18HPV groups.

- hrHPV test positivity (hrHPV positive tests/HPV tests).

- Cytology test positivity (abnormal cytology tests/ cytology tests).

- Cytology and hrHPV cotesting positivity (women with hrHPV-positive tests or abnormal cytology tests/women who underwent cytology and hrHPV cotesting).

- Concordance rate of the colposcopic impression (women with colposcopic impression met CDB pathology/women with colposcopic impression).

\section{Results}

The numbers of cytology and hrHPV screenings performed in 2019 were 109,009 and 76,189, respectively. In Yangpu district, a total of 26,432 colposcopies were performed. Based on the histologic results, $8.1 \%$ were VaIN+. As the largest OBGYN referral centre in China, most patients were referred to our hospital with abnormal cervical screening results; therefore, the exact prevalence of vaginal lesions cannot be calculated.

A total of 2,131 women were included, as follows: 1835 (86.1\%) with VaIN 1; 268 (12.6\%) with VaIN 2/3; and $28(1.3 \%)$ with vaginal cancer. The clinical characteristics of women with VaIN and vaginal cancer are presented in Table 1. The median age was 48 years (IQR, 3556 years). Women with VaIN 1 were younger than women with VaIN 2/3 and vaginal cancer (47 years vs 52 and 53.5 years; $\mathrm{P}<0.001)$. Approximately 1260 (59.1\%) patients were diagnosed with only vaginal lesions. Among 871 patients with concomitant lesions, $81.3 \%$ had cervical lesions, $10.3 \%$ had vulvar lesions, and $8.4 \%$ had both. The rates of concomitant lesions with VaIN 1 and VaIN $2 / 3$ were similar ( $41.3 \%$ vs $39.6 \%$ ); however, the rate was lowest with vaginal cancer $(25.0 \% ; \mathrm{P}=0.19)$.

Twenty-eight patients had vaginal cancer. Only half of them had HSIL+ cytology results, whereas $84.6 \%(22 / 26)$ had positive hrHPV results and 75.0\% (15/20) had HPV 16 infection among positive hrHPV genotyping tests. Approximately $92.8 \%$ of vaginal cancer diagnoses were consistent with the colposcopic impressions. Seventeen patients had a history of hysterectomy because of cervical lesions $(n=16)$ or endometrial cancer $(n=1)$.

\section{Cytology and hrHPV Screening}

The overall positivity was highest in cytology and hrHPV cotesting, followed by hrHPV testing alone and cytology alone $(96.5 \%, 85.4 \%$ and $69.0 \%$; $\mathrm{P}<0.001)$.

A total of 2123 cytology results were available. The detailed cytology reports are presented in Table 2. The cytology test positivity for vaginal cancer and VaIN 2/3 was higher than that for VaIN 1 (78.6\% and 79.9\% vs $67.3 \%$; $\mathrm{P}<0.001)$. Based on the cytology tests, cytology test positivity was highest in patients who had undergone hysterectomy and lowest in patients without cervical lesions in their intact uterus $(76.2 \%$ and $61.5 \%$; $\mathrm{P}<$ 0.001). Concomitant cervical lesions also increased the cytology test positivity to $75.0 \%$ (Figure 1 ).

A total of $2120 \mathrm{hrHPV}$ results were available for 2131 women with VaIN or vaginal cancer. Of these, 143 underwent hybrid capture- 2 testing, 581 underwent Cobas testing, and 1396 underwent BMRT genotyping. The hrHPV test positivity was highest for VaIN $2 / 3$ at $92.0 \%$, which was significantly higher than those for VaIN 1 (84.4\%) and vaginal cancer $(84.6 \%)$. hrHPV test positivity was slightly higher among patients after hysterectomy than among patients without cervical lesions, but no significant difference was found $(85.0 \%$ vs $82.5 \%$; $\mathrm{P}=0.289)$.

Among hrHPV-positive patients using BMRT genotyping, HPV 16 was the most prevalent HPV type, with a positivity of $26.5 \%$, followed by HPV $52(24.3 \%)$, HPV 58 (22.5\%) and HPV 56 (12.6\%). The detailed ranking of infected hrHPV genotypes among VaIN 1, VaIN 2/3, and vaginal cancer is illustrated in Figure 2. More than half of the patients with VaIN 2/3 (64.5\%) and vaginal cancer $(88.9 \%)$ were infected with HPV 16, while 
Table I Clinical Characteristics of 2131 Women with VaIN and Vaginal Cancer

\begin{tabular}{|c|c|c|c|c|}
\hline Characteristics & ValN I & ValN $2 / 3$ & Vaginal Cancer & $P$-value \\
\hline Age(y), Median (IQR) & $47(34-56)$ & $52(42-6 I)$ & $53.5(40-62.5)$ & $<0.01$ \\
\hline \multicolumn{5}{|l|}{ Cytology, n (\%) } \\
\hline NLIM & $599(32.7)$ & $53(20.1)$ & $6(2 \mid .4)$ & \\
\hline ASCUS or LSIL & $1147(62.7)$ & $132(50.0)$ & $8(28.6)$ & \\
\hline AGC, ASC-H or HSIL+ & $85(4.6)$ & $79(29.9)$ & $14(50.0)$ & \\
\hline hrHPV infection, n (\%) & & & & $<0.01$ \\
\hline No & $285(15.6)$ & $21(8.0)$ & $4(15.4)$ & \\
\hline Yes & $1545(84.4)$ & $243(92.0)$ & $22(84.6)$ & \\
\hline Cotesting sensitivity, n (\%) & & & & 0.02 \\
\hline No & $74(4.1)$ & I (0.4) & I (3.8) & \\
\hline Yes & $1752(95.9)$ & $261(99.6)$ & $25(96.2)$ & \\
\hline Colposcopy impression, n (\%) & & & & $<0.01$ \\
\hline Normal & $225(12.3)$ & $8(3.0)$ & $0(0.0)$ & \\
\hline ValN I & 1459 (79.5) & $103(38.4)$ & I (3.6) & \\
\hline ValN $2 / 3$ & $|5|(8.2)$ & $146(54.5)$ & I (3.6) & \\
\hline Vaginal cancer & $0(0.0)$ & II (4.I) & $26(92.8)$ & \\
\hline Concomitant lesion, n (\%) & & & & 0.19 \\
\hline No & $1077(58.7)$ & $162(60.4)$ & $21(75.0)$ & \\
\hline Yes & $758(4 \mid .3)$ & $106(39.6)$ & $7(25.0)$ & \\
\hline Hysterectomy, n (\%) & & & & $<0.01$ \\
\hline No & $1590(86.6)$ & I $58(59.0)$ & II (39.3) & \\
\hline Yes & $245(13.4)$ & $110(41.0)$ & $17(60.7)$ & \\
\hline
\end{tabular}

Note: hrHPV including HPV 16, 18, 31, 33, 35, 39, 45, 51, 52, 56, 58, 59, 66, 68.

Abbreviations: VaIN, vaginal intraepithelial neoplasia; NLIM, negative for intraepithelial lesion or malignancy; ASCUS, atypical squamous cells of undetermined significance; ASC-H, atypical squamous cells, cannot exclude high-grade squamous intraepithelial lesion; LSIL, low-grade squamous intraepithelial lesion; HSIL, high-grade squamous intraepithelial lesion; AGC, atypical glandular cells; IQR, interquartile range.

HPV $52(25.9 \%)$ and HPV $58(24.1 \%)$ were more prevalent than HPV $16(20.4 \%)$ in patients with VaIN 1.

Regardless of the degree of vaginal lesions and the hysterectomy history, the cytology and hrHPV cotesting positivity was above $95 \%$. There was no significant difference in cotesting positivity between patients after hysterectomy and patients without cervical lesions.

Seventy-six patients had negative cotesting test. The indications for colposcopy were as follows: abnormal genital tract bleeding or unexplained cervicovaginal discharge; first follow-up after cervical conization; and first follow-up after hysterectomy indicated for cervical cancer or HSIL. Among them, one patient was confirmed to have $\mathrm{VaIN} 2 / 3$ and another patient was confirmed to have vaginal cancer. Both underwent hysterectomy for cervical HSIL.

\section{Colposcopic Impression}

All of the colposcopy records were available. The concordance rates of the colposcopic impression were $79.5 \%$
(1459/1835), 54.5\% (146/243), and 92.8\% (26/28) for patients with VaIN 1, VaIN 2/3, and vaginal cancer, respectively. Approximately $41.4 \%$ of VaIN $2 / 3$ patients were underestimated by colposcopic impression.

\section{Hysterectomy History}

All 372 women with a history of hysterectomy underwent either total or radical hysterectomy. Among them, 348 hysterectomy indications were available (Table 3). Overall, the most common indications were precancerous cervical lesions (44.8\%) and overt cancer (36.2\%). For patients with vaginal cancer, $94.5 \%(16 / 17)$ of the patients underwent hysterectomy for cervical lesions, while the other hysterectomy indication was endometrial cancer. Two patients (aged 38 and 46 years) underwent hysterectomy and partial vaginectomy because of vaginal cancer. Both of them had VaIN 1 diagnosed several years after surgery.

The proportions of VaIN 2/3 and vaginal cancer were significantly higher in patients who had undergone 
Table 2 Detailed Cytology Results of 2123 Women with ValN and Vaginal Cancer

\begin{tabular}{|c|c|c|c|c|c|c|c|c|c|}
\hline Cytology Test Results & NLIM & ASCUS & LSIL & HSIL & ASC-H & SCC & AGC & Total & Positivity \\
\hline ValN I & 599 & 425 & 722 & 59 & 23 & I & 2 & $|83|$ & $67.3 \%$ \\
\hline VaIN I with uterus & 534 & 361 & 624 & 45 & 19 & I & 2 & 1586 & $66.3 \%$ \\
\hline Without cervical lesion & 355 & 214 & 315 & 12 & 10 & 0 & I & 907 & $60.9 \%$ \\
\hline Concomitant cervical lesion & 179 & 147 & 309 & 33 & 9 & I & I & 679 & $73.6 \%$ \\
\hline ValN I after hysterectomy & 65 & 64 & 98 & 14 & 4 & 0 & 0 & 245 & $73.5 \%$ \\
\hline ValN $2 / 3$ & 53 & 53 & 79 & 60 & 14 & 4 & I & 264 & $79.9 \%$ \\
\hline ValN $2 / 3$ with uterus & 35 & 31 & 46 & 34 & 7 & 3 & 0 & 156 & $77.6 \%$ \\
\hline Without cervical lesion & 20 & 13 & 22 & 7 & I & 2 & 0 & 65 & $69.2 \%$ \\
\hline Concomitant cervical lesion & 15 & 18 & 24 & 27 & 6 & I & 0 & 91 & $83.5 \%$ \\
\hline ValN $2 / 3$ after hysterectomy & 18 & 22 & 33 & 26 & 7 & I & I & 108 & $83.3 \%$ \\
\hline Vaginal cancer & 6 & 6 & 2 & 10 & I & 3 & 0 & 28 & $78.6 \%$ \\
\hline Vaginal cancer with uterus & 1 & 4 & I & 4 & 0 & I & 0 & 11 & $90.9 \%$ \\
\hline Without cervical lesion & I & 2 & 0 & 1 & 0 & 0 & 0 & 4 & $75.0 \%$ \\
\hline Concomitant cervical lesion & 0 & 2 & I & 3 & 0 & I & 0 & 7 & $100.0 \%$ \\
\hline Vaginal cancer after hysterectomy & 5 & 2 & I & 6 & I & 2 & 0 & 17 & $70.6 \%$ \\
\hline Total & 658 & 803 & 129 & 484 & 38 & 8 & 3 & 2123 & $69.0 \%$ \\
\hline
\end{tabular}

Notes: Positivity, abnormal cytology tests /cytology tests.

Abbreviations: VaIN, vaginal intraepithelial neoplasia; NLIM, negative for intraepithelial lesion or malignancy; ASCUS, atypical squamous cells of undetermined significance; ASC-H, atypical squamous cells, cannot exclude high-grade squamous intraepithelial lesion; LSIL, low-grade squamous intraepithelial lesion; HSIL, high-grade squamous intraepithelial lesion; AGC, atypical glandular cells. SCC, squamous cell carcinoma.

hysterectomy. The median age of patients who had undergone hysterectomy was 10 years older than that of patients who had not $(\mathrm{P}<0.001)$. The sensitivity of cytology was higher in patients who had undergone hysterectomy $(\mathrm{P}=$ 0.001 ), whereas the sensitivity values of hrHPV and concurrent cytology/hrHPV testing were not significantly different $(\mathrm{P}=0.87$ and $\mathrm{P}=0.88$, respectively).

\section{Discussion}

Compared with CIN, VaIN+ has not been sufficiently researched. The incidence of VaIN (0.2-0.3 per 100,000 women) in most studies was reported by epidemiological research involving large samples published in 1977, which was almost 50 years ago. ${ }^{19}$ However, since then, developments in medical instruments and medical care

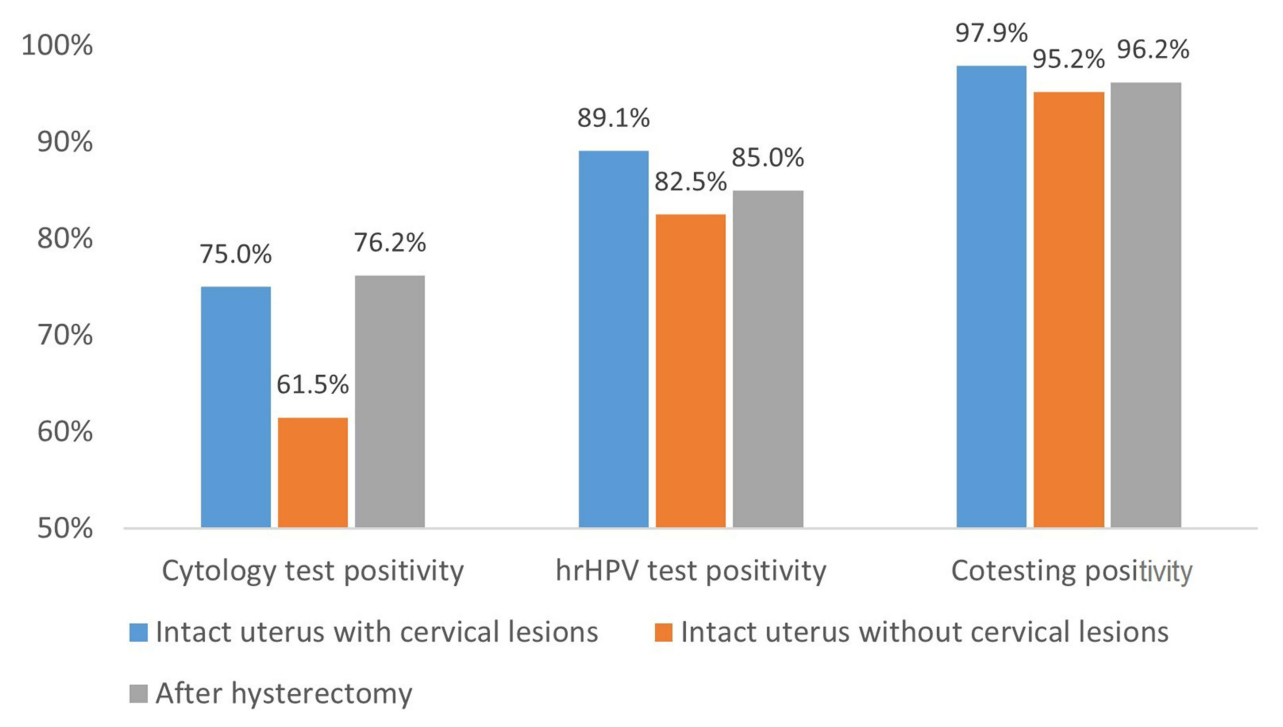

Figure I The positivity of cytology, hrHPV and cotesting in ValN and vaginal cancer.

Note: Cytology test positivity, abnormal cytology tests/cytology tests. hrHPV test positivity, hrHPV positive tests/HPV tests. Cotesting positivity, women with hrHPV positive tests or abnormal cytology tests/women performed cytology and hrHPV cotesting.

Abbreviation: ValN, vaginal intraepithelial neoplasia. 


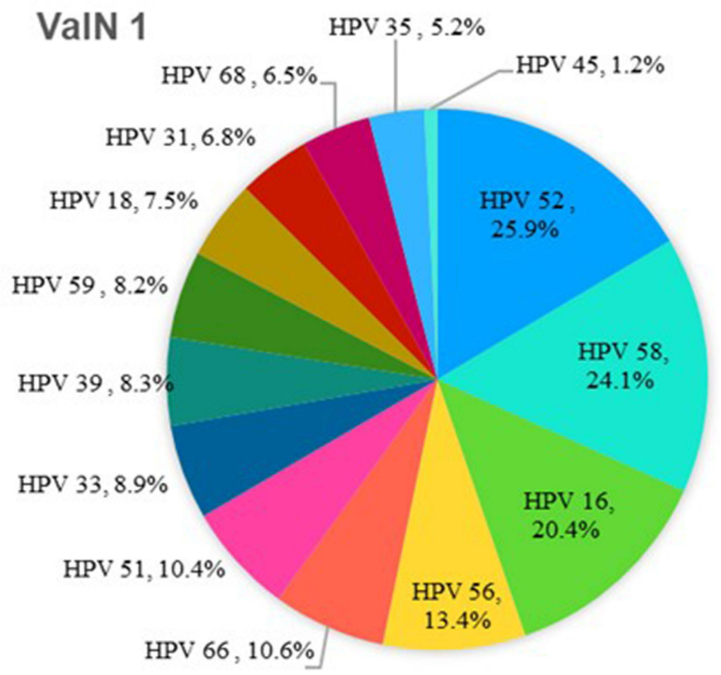

Vaginal cancer

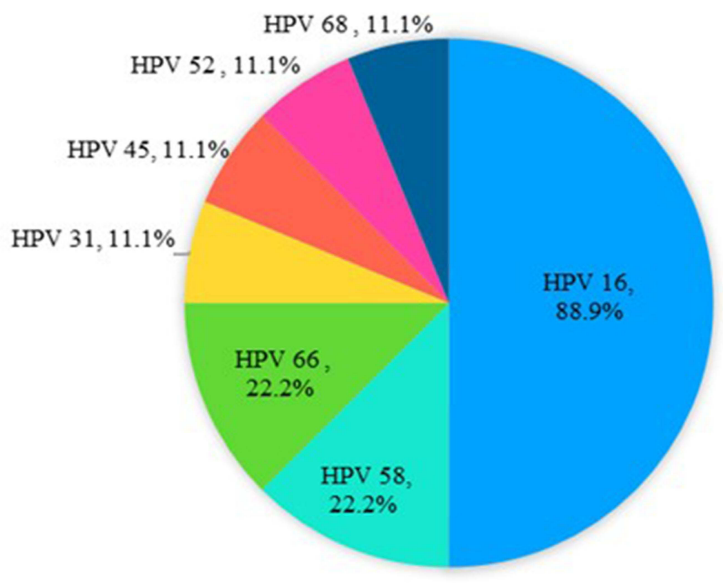

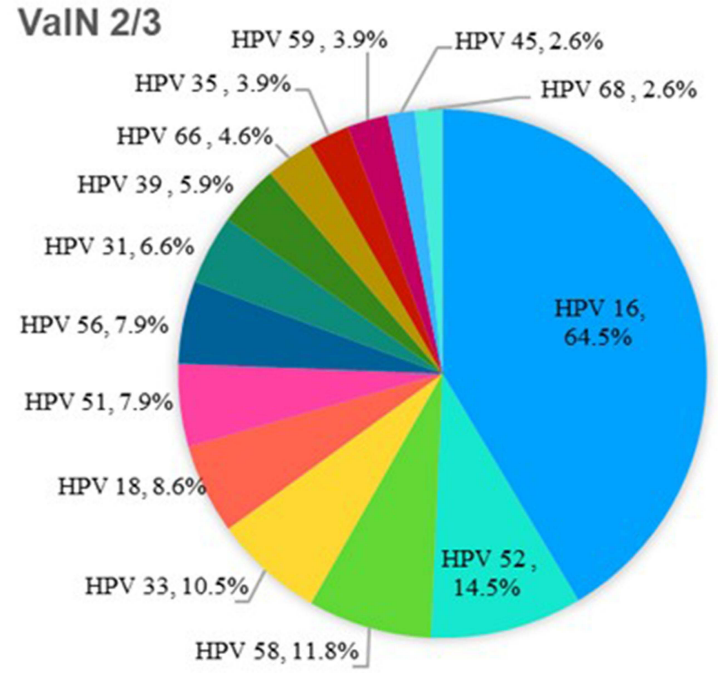

HPV 58, $11.8 \%$
Figure 2 Distribution of hrHPV in ValN and vaginal cancer.

Note: hrHPV including HPV 16, I8, 31, 33, 35, 39, 45, 5I, 52, 56, 58, 59, 66, 68. Abbreviation: ValN, vaginal intraepithelial neoplasia.

have improved. We previously reported 16,732 women diagnosed with CIN (83.99\%; 14,053/16,732), VaIN $(11.49 \% ; 1923 / 16,732)$, and $\operatorname{VIN}(4.52 \% ; 756 / 16,732)$ at our hospital between 2013 and 2015. ${ }^{11}$ The annual number of VaIN+ cases in 2019 was 2131, which is approximately sixfold that of 2013 . Hence, VaIN+ is not a rare disease during colposcopy. One reason for the rapidly increased number of diagnosed cases could be that the total number of CDB increased annually at our hospital. Furthermore, increasing attention has been focused on VaIN + by colposcopists. Therefore, the current actual prevalence of VaIN + might be much higher than what has been reported.

With a rapid increase in the prevalence of $\mathrm{VaIN}+$, there are no guidelines for vaginal lesion screening. Most VaIN+ was found at the time of cervical screening. However, since we perform cytology screening on the cervical surface, there is doubt whether it is enough for the detection of VaIN+. Few studies have explored the value of cytology for VaIN or vaginal cancer. ${ }^{20-22}$ A meta-analysis by Khan et al indicated that for patients with VaIN 2/3 who have undergone hysterectomy, the cytology test positivity was $83 \%$ and the positive predictive value ranged from $0 \%$ to $14 \%{ }^{23}$ Our study showed that cytology test positivity was $79.9 \%$ for VaIN $2 / 3$ and $78.6 \%$ for vaginal cancer; however, it was only $67.6 \%$ for VaIN 1 . Despite differences in cytology results, the positivity of cotesting with and without hysterectomy was more than $95 \%$.

Studies have demonstrated that various HPV types might have different roles in the risk of CIN and cervical 
Table 3 Indications and Rates of Previous Hysterectomy in 348 ValN and Vaginal Cancer Patients

\begin{tabular}{|c|c|c|c|c|c|c|c|c|}
\hline \multirow[t]{2}{*}{ Indications of Hysterectomy } & \multicolumn{2}{|c|}{ ValN I } & \multicolumn{2}{|c|}{ ValN $2 / 3$} & \multicolumn{2}{|c|}{ Vaginal Cancer } & \multicolumn{2}{|c|}{ Total } \\
\hline & $\mathbf{n}$ & $\%$ & $\mathbf{n}$ & $\%$ & $\mathbf{n}$ & $\%$ & $\mathbf{n}$ & $\%$ \\
\hline \multicolumn{9}{|l|}{ Cervical lesions } \\
\hline Cervical cancer & 81 & 35.5 & 33 & 32.0 & 12 & 70.6 & 126 & 36.2 \\
\hline Cervical precancer lesion & 95 & 41.7 & 57 & 55.3 & 4 & 23.5 & 156 & 44.8 \\
\hline \multicolumn{9}{|l|}{ Noncervical lesions } \\
\hline Vaginal cancer* & 2 & 0.9 & 0 & 0.0 & 0 & 0.0 & 2 & 0.6 \\
\hline Endometrial cancer & 6 & 2.6 & $\mathrm{I}$ & 1.0 & I & 5.9 & 8 & 2.3 \\
\hline Ovarian cancer & 0 & 0.0 & I & 1.0 & 0 & 0.0 & I & 0.3 \\
\hline Benign disease $^{\wedge}$ & 44 & 19.3 & 11 & 10.7 & 0 & 0.0 & 55 & 15.8 \\
\hline Total & 228 & 100.0 & 103 & 100.0 & 17 & 100.0 & 348 & 100.0 \\
\hline
\end{tabular}

Notes: *One patient had hysterectomy and follow-up radiotherapy for vaginal primary mucinous adenocarcinoma 5 years ago. The other patient was diagnosed with vaginal squamous cell carcinoma and underwent surgery 3 years ago. ^Benign diseases including myoma, adenomyosis, uterine prolapse, endometrial hyperplasia, obstetrics reasons. Abbreviation: ValN, vaginal intraepithelial neoplasia.

cancer progression. ${ }^{24}$ However, few studies have focused on the role of HPV infection in VaIN+. Only 3 studies involving more than 100 cases analyzed the HPV genotyping distribution in VaIN+. ${ }^{12,14,15}$ Alemany et al performed a worldwide cross-sectional study of 189 VaIN 2/3 cases and 408 vaginal cancer cases in 31 countries from 1986 to 2011 and reported that the HPV prevalence was $96 \%$ with VaIN 2/3 lesions and 74\% with vaginal cancer. ${ }^{15}$ Bertoli et al reported that the pooled HPV prevalence was $85.2 \%$ with VaIN and $66.7 \%$ with vaginal cancer. ${ }^{25}$ Furthermore, the most predominant HPV type was found to be HPV 16, followed by HPV 33 in VaIN and HPV 18 in vaginal cancer. Our study included 2131 Chinese women with VaIN+; hrHPV test positivity was $84.4 \%$ for patients with VaIN 1, 92.0\% for patients with VaIN 2/3, and $84.6 \%$ for patients with vaginal cancer. Similarly, HPV 16 was the predominant genotype; it was found in $64.5 \%$ of VaIN $2 / 3$ cases and $88.9 \%$ of vaginal cancer cases among hrHPV-positive patients. HPV 52, HPV 58, and HPV 18 were more prevalent than HPV 33 in patients with VaIN2/3, and HPV 58 and HPV 66 were more prevalent than HPV 18 in patients with vaginal cancer. In contrast, HPV 52 (25.9\%) was the predominant type found in patients with VaIN 1, followed by HPV 58 (24.1\%) and HPV 16 (20.4\%).

$\mathrm{CDB}$ is critical for detecting precancer and cancer of the cervix and vagina. Accumulating studies have indicated the poor accuracy and specificity of colposcopic impressions when diagnosing cervical neoplasia. ${ }^{26,27}$ Because of its rarity, the accuracy of colposcopic examinations for detecting $\mathrm{VaIN}+$ remains unclear. Only one study of VaIN revealed that the colposcopy impression accuracy was $52.2 \%$, with higher accuracy observed for VaIN $2 / 3$ patients $(82.5 \%)$ than for VaIN 1 patients (45.0\%). Furthermore, the detection rate of VaIN 2/3 may be improved if testing is performed by a highly experienced practitioner. ${ }^{13}$ Our previous study of vaginal cancer demonstrated that colposcopic examination is not only pivotal for the evaluation of abnormal cytology/ hrHPV tests after hysterectomy but also decision-making for vaginectomy in the detection of early cancer. ${ }^{28}$ In the present study, the overall agreement of colposcopy and biopsy results was $76.5 \%$; the agreement of colposcopy and biopsy results for VaIN 1, VaIN 2/3 and vaginal cancer was $79.5 \%, 54.5 \%$ and $92.8 \%$, respectively $(\mathrm{P}<0.001)$.

Various studies have shown that a history of CIN 2/3 and cervical cancer was strongly associated with VaIN+. Cervical precancer/cancer patients who have undergone hysterectomy are more likely to be diagnosed with subsequent VaIN+ than women without these risk factors who have undergone hysterectomy. ${ }^{29,30}$ Therefore, the 2019 ASCCP Risk-Based Management Consensus Guidelines for Abnormal Cervical Cancer Screening Tests and Cancer Precursors recommend that women who have undergone total hysterectomy for cervical precancer or invasive cervical cancer should undergo surveillance for at least 25 years. $^{31}$ In $81.0 \%$ of hysterectomy cases, the indication for hysterectomy was cervical precancer/cancer. For patients with vaginal cancer, $23.5 \%$ had a history of cervical precancer and $70.6 \%$ had a history of cervical cancer. These results may be partially explained by the persistence of hrHPV infection in women with prevalent CIN, which is 
possibly lifelong. Vaginal cancer might develop from VaIN $2 / 3$ before or after hysterectomy with persistent hrHPV infection. However, VaIN 1 is merely an expression of HPV infection and is often transient. Thus, women who underwent hysterectomy for cervical precancer/cancer were at high risk for VaIN $2 / 3+$. Therefore, in our study, for patients who underwent hysterectomy, the rate of VaIN $2 / 3$ vaginal lesions was approximately threefold that of patients who did not undergo hysterectomy $(29.5 \%$ vs $9.0 \%$; $\mathrm{P}<$ 0.001). Careful examination of the vagina during colposcopy before hysterectomy is crucial to exclude VaIN concurrent with cervical precancer/cancer.

The incidence of vaginal cancer was found to be highly age-related in one large study. ${ }^{32}$ In our study, the mean age of patients with vaginal cancer was 52.5 years, which was 2 years older than those with VaIN 2/3 and almost 7 years older than those with VaIN 1 . This suggests that it may take years to progress to VaIN $2 / 3$ and vaginal cancer.

To best of our knowledge, this is the largest retrospective study to investigate cervical screening history, including cytology results, hrHPV results, and colposcopy results, of VaIN + patients in China. Our data demonstrated that VaIN is not a rare disease. It renewed the acknowledgement of VaIN and attracted the attention of clinical examinations. However, there are two limitations. First, our survey was conducted at a single centre in China, so selection bias is inevitable Second, the patients in our hospital were referred from other hospitals in all of China; thus, we were unable to calculate the prevalence of VaIN in the population.

\section{Conclusion}

As the largest OBGYN referral centre in China, the prevalence of VaIN+ has been increasing rapidly since 2013 . Although cytology was sensitive $(67.5 \%-76.2 \%)$ for detecting vaginal lesions regardless of hysterectomy, cytology combined with hrHPV improve detection accuracy up to $95 \%$ in both groups.

\section{Data Sharing Statement}

Data in this study are available from Dr Qing Cong upon reasonable request.

\section{Ethical Approval}

Approval was obtained from the institutional review board of the Obstetrics and Gynecology Hospital of Fudan University before data extraction was performed. All patients acquired informed consent.

\section{Funding}

No external funding from private or public organizations were received for the conduct of this study or the preparation of this manuscript.

\section{Disclosure}

The authors declare that there is no conflict of interest regarding the publication of this paper.

\section{References}

1. Bertoli HK, Thomsen LT, Iftner T, et al. Risk of vulvar, vaginal and anal high-grade intraepithelial neoplasia and cancer according to cervical human papillomavirus (HPV) status: a population-based prospective cohort study. Gynecol Oncol. 2020;157(2):456-462. PMID: 32008794. doi:10.1016/j.ygyno.2020.01.030

2. Bogani G, Martinelli F, Ditto A, et al. Human papillomavirus (HPV) persistence and HPV 31 predict the risk of recurrence in high-grade vaginal intraepithelial neoplasia. Eur J Obstet Gynecol Reprod Biol. 2017;210:157-165. PMID: 28039759. doi:10.1016/j.ejogrb.20 16.12 .020

3. De Vuyst H, Clifford GM, Nascimento MC, et al. Prevalence and type distribution of human papillomavirus in carcinoma and intraepithelial neoplasia of the vulva, vagina and anus: a meta-analysis. Int J Cancer. 2009;124(7):1626-1636. PMID: 19115209. doi:10.10 $02 / \mathrm{ijc} .24116$

4. Cong Q, Song Y, Wang Q, et al. A retrospective study of cytology, high-risk HPV, and colposcopy results of vaginal intraepithelial neoplasia patients. Biomed Res Int. 2018;2018:5894801. PMID: 29854768; PMCID: PMC5964579. doi:10.1155/2018/5894801

5. Saslow D, Solomon D, Lawson HW, et al. American Cancer Society, American Society for Colposcopy and Cervical Pathology, and American Society for Clinical Pathology screening guidelines for the prevention and early detection of cervical cancer. Am J Clin Pathol. 2012;137(4):516-542. PMID: 22431528. doi:10.1309/AJCP TGD94EVRSJCG

6. Darragh TM, Colgan TJ, Cox JT, et al. The Lower Anogenital Squamous Terminology Standardization Project for HPV-Associated Lesions: background and consensus recommendations from the College of American Pathologists and the American Society for Colposcopy and Cervical Pathology. Arch Pathol Lab Med. 2012;136(10):1266-1297. PMID: 22742517. doi:10.5858/arpa. LGT200570

7. Daling JR, Madeleine MM, Schwartz SM, et al. A population-based study of squamous cell vaginal cancer: HPV and cofactors. Gynecol Oncol. 2002;84(2):263-270. PMID: 11812085. doi:10.1006/gyno.20 01.6502

8. Field A, Bhagat N, Clark S, et al. Vaginal intraepithelial neoplasia: a retrospective study of treatment and outcomes among a cohort of UK women. J Low Genit Tract Dis. 2020;24(1):43-47. PMID: 31860574. doi:10.1097/LGT.0000000000000502

9. Pan J, Kavanagh K, Cuschieri K, et al. Increased risk of HPV-associated genital cancers in men and women as a consequence of pre-invasive disease. Int $J$ Cancer. 2019;145 (2):427-434. PMID: 3065018. doi:10.1002/ijc.32126

10. Edgren G, Sparén P. Risk of anogenital cancer after diagnosis of cervical intraepithelial neoplasia: a prospective population-based study. Lancet Oncol. 2007; (4):311-316. PMID: 17395104. doi:10.1016/S1470-2045(07)70043-8

11. Cong Q, Wang Q, Gao SJ, et al. Detection trend of vaginal intraepithelial neoplasia diagnosed by colposcopy guided biopsy from 2013 to 2015. Zhonghua Fu Chan Ke Za Zhi. 2017;52(4):239-243; PMID: 28441839. doi: 10.3760/cma.j.issn.0529-567X.2017.04.005 
12. Chao A, Chen TC, Hsueh C, et al. Human papillomavirus in vaginal intraepithelial neoplasia. Int $J$ Cancer. 2012;131(3):E259-E268. PMID: 22095387. doi:10.1002/ijc. 27354

13. Stuebs FA, Koch MC, Mehlhorn G, et al. Accuracy of colposcopic findings in detecting vaginal intraepithelial neoplasia: a retrospective study. Arch Gynecol Obstet. 2020;301(3):769-777. PMID: 31993733. doi:10.1007/s00404-020-05441-5

14. Zhang J, Chang X, Qi Y, et al. A retrospective study of 152 women with vaginal intraepithelial neoplasia. Int J Gynaecol Obstet. 2016;133 (1):80-83. PMID: 26797205. doi:10.1016/j.ijgo.2015.08.014

15. Alemany L, Saunier M, Tinoco L, et al. Large contribution of human papillomavirus in vaginal neoplastic lesions: a worldwide study in 597 samples. Eur $J$ Cancer. 2014;50(16):2846-2854. PMID: 25155250. doi:10.1016/j.ejca.2014.07.018

16. Sinno AK, Saraiya M, Thompson TD, et al. Human papillomavirus genotype prevalence in invasive vaginal cancer from a registry-based population. Obstet Gynecol. 2014;123(4):817-821. PMID: 24785610; PMCID: PMC4009490. doi:10.1097/AOG.0000000000000171

17. Bornstein J, Bentley J, Bösze P, et al. 2011 colposcopic terminology of the International Federation for Cervical Pathology and Colposcopy. Obstet Gynecol. 2012;120(1):166-172. PMID: 22914406. doi:10.1097/AOG.0b013e318254f90c

18. Zhou Q, Zhang F, Sui L, et al. Application of 2011 International Federation for Cervical Pathology and Colposcopy Terminology on the Detection of Vaginal Intraepithelial Neoplasia. Cancer Manag Res. 2020;12:5987-5995.

19. Henson D, Tarone R. An epidemiologic study of cancer of the cervix, vagina, and vulva based on the Third National Cancer Survey in the United States. Am J Obstet Gynecol. 1977;129(5):525-532. PMID: 199064.

20. So KA, Hong JH, Hwang JH, et al. The utility of the human papillomavirus DNA load for the diagnosis and prediction of persistent vaginal intraepithelial neoplasia. J Gynecol Oncol. 2009;20(4):232-237. PMID: 20041100; PMCID: PMC2799022. doi:10.3802/jgo.2009.20.4.232

21. Frega A, French D, Piazze J, et al. Prediction of persistent vaginal intraepithelial neoplasia in previously hysterectomized women by high-risk HPV DNA detection. Cancer Lett. 2007;249(2):235-241. PMID: 17070990. doi:10.1016/j.canlet.2006.09.003

22. Bansal M, Austin RM, Zhao C. Correlation of histopathologic follow-up findings with vaginal human papillomavirus and low-grade squamous intraepithelial lesion Papanicolaou test results. Arch Pathol Lab Med. 2011;135(12):1545-1549. PMID: 22129181. doi:10.5858/arpa.2010-0658-OA
23. Khan MJ, Massad LS, Kinney W, et al. A common clinical dilemma: management of abnormal vaginal cytology and human papillomavirus test results. J Low Genit Tract Dis. 2016;20(2):119-125. PMID: 26901279. doi:10.1097/LGT.0000000000000185

24. Thomsen LT, Frederiksen K, Munk C, et al. Long-term risk of cervical intraepithelial neoplasia grade 3 or worse according to high-risk human papillomavirus genotype and semi-quantitative viral load among 33,288 women with normal cervical cytology. Int $J$ Cancer. 2015;137 (1):193-203. PMID: 25471319. doi:10.1002/ijc.29374

25. Bertoli HK, Rasmussen CL, Sand FL, et al. Human papillomavirus and p16 in squamous cell carcinoma and intraepithelial neoplasia of the vagina. Int $J$ Cancer. 2019;145(1):78-86. PMID: 30561092. doi:10.1002/ijc.32078

26. Mustafa RA, Santesso N, Khatib R, et al. Systematic reviews and meta-analyses of the accuracy of HPV tests, visual inspection with acetic acid, cytology, and colposcopy. Int J Gynaecol Obstet. 2016;132 (3):259-265. PMID: 26851054. doi:10.1016/j.ijgo.2015.07.024

27. Brown BH, Tidy JA. The diagnostic accuracy of colposcopy - A review of research methodology and impact on the outcomes of quality assurance. Eur J Obstet Gynecol Reprod Biol. 2019;240:182-186. PMID: 31302386. doi:10.1016/j.ejogrb.2019.07.003

28. Cong Q, Fu Z, Zhang D, et al. Importance of colposcopy impression in the early diagnosis of posthysterectomy vaginal cancer. J Low Genit Tract Dis. 2019;23(1):13-17. PMID: 30418351; PMCID: PMC6325769. doi:10.1097/LGT.0000000000000446

29. Tidy J. The risk of vaginal cancer is associated with a history of cervical neoplasia. BJOG. 2020;127(4):455. PMID: 31863542. doi:10.1111/147-0528.16065

30. Li Z, Barron S, Hong W, et al. Surveillance for recurrent cancers and vaginal epithelial lesions in patients with invasive cervical cancer after hysterectomy: are vaginal cytology and high-risk human papillomavirus testing useful? Am J Clin Pathol. 2013;140(5):708-714. PMID: 24124151. doi:10.1309/AJCPH4AFSZHU8EKK

31. Perkins RB, Guido RS, Castle PE, et al. 2019 ASCCP risk-based management consensus guidelines for abnormal cervical cancer screening tests and cancer precursors. J Low Genit Tract Dis. 2020;24(2):102-131. PMID: 32243307; PMCID: PMC7147428. doi:10.1097/LGT.0000000000000525

32. Strander B, Andersson-Ellström A, Milsom I, et al. Long term risk of invasive cancer after treatment for cervical intraepithelial neoplasia grade 3: population based cohort study. BMJ. 2007;335(7629):1077. PMID: 17959735; PMCID: PMC2094166. doi:10.1136/bmj.39363.471806.BE
Cancer Management and Research

\section{Publish your work in this journal}

Cancer Management and Research is an international, peer-reviewed open access journal focusing on cancer research and the optimal use of preventative and integrated treatment interventions to achieve improved outcomes, enhanced survival and quality of life for the cancer patient.
The manuscript management system is completely online and includes a very quick and fair peer-review system, which is all easy to use Visit http://www.dovepress.com/testimonials.php to read real quotes from published authors. 